\title{
Le sacrifice, le mariage, la mort et la folie chez les Thonga
}

Sacrifice, marriage, death and madness among the Thonga

Luc de Heusch

\section{OpenEdition}

1 Journals

Édition électronique

URL : http://journals.openedition.org/span/362

DOI : 10.4000/span.362

ISSN : 2268-1558

Éditeur

École pratique des hautes études. Sciences humaines

\section{Édition imprimée}

Date de publication : 1 septembre 1978

Pagination : 59-85

ISSN : 0294-7080

\section{Référence électronique}

Luc de Heusch, «Le sacrifice, le mariage, la mort et la folie chez les Thonga », Systèmes de pensée en

Afrique noire [En ligne], 3 | 1978, mis en ligne le 06 juin 2013, consulté le 02 mai 2019. URL : http:// journals.openedition.org/span/362 ; DOI : 10.4000/span.362 
P R É S E N T A T I O N

par Lue de Heusch

Ce second cahier consacré aux systèmes sacrificiels comprend deux parties. Nous souhaitons d'abord verser quelques éléments nouveaux au dossier africain présenté par le Laboratoire 221 dans une publication précédente (1). Nous ne prétendons pas clore un inventaire toujours ouvert. L'ethnographie africaine n'a que trop souvent jetê un regard distrait sur la basse-cour, les chèvres, les moutons, sinon sur les boeufs. Elle a négligé le fait que la pensée classificatoire opérait aussi des choix préméditês dans le petit monde compact des animaux de village, unis à $I^{\prime}$ homme par des liens étroits d'ordre divers, qui n'ont que rarement été dêcrits systématiquement : économiques, symboliques, rituels.

N'est-ce pas cependant au centre de ces réseaux que le sacrifice prend figure?

Que l'enquête soit appelée à s'affiner, à épouser les innombrables nuances du code sacrificiel, de sa pratique et de sa finalité, souvent occultée par des métaphores de caractère alimentaire, la dernière enquête de Michel Cartry en Haute-Volta le démontre de manière exemplaire. Cartry approfondit ici l'analyse déjà esquissée dans le premier Cahier.

1. Le sacrifice, I, 1976. 
Il met en lumière le rôle fondamental de la géomancie comme "ordinateur" du sacrifice en pays gourmantché. Il dégage les relations sēmiologiques et topologiques très complexes qui se nouent entre les "paroles de la terre", leur inscription sur un morceau de calebasse et 1 'animal domestique, immolé en même temps qu'est dêtruit 1'objet prêcêdent. L"écriture" divinatoire, indissociable du sacrifice gourmantché, n'utilise pas moins de 19 signes distincts pour signifier différentes variétés de poulets et certains d'entre eux relèvent d'un bestiaire fantasmagorique. D'ailleurs 1 'animal apporté sur le lieu du sacrifice "n'est pas nëcessairement porteur des caractëristiques évoquées par le signe gravê" ; celui-ci donne lieu à des jeux de mots subtils qui permettent au sacrificateur d'exprimer la finalité du rite en se livrant à une véritable "lecture" du corps même du poulet. La mise en jeu des signes inscrits sur le morceau de calebasse, puis détruits, impose donc à l'animal "une double transformation de statut". Le problème classique de la substitution des animaux doit être interprété dans cette perspective : dans le système gourmantché, c'est la parole du sacrificateur qui rend la victime porteuse des caractéristiques "lues sur le signe". Et c'est bien sur des signes, transmis à Dieu par 1'intermédiaire d'une puissance fétichisée (buli), que la pensée sacrificielle opère.

Attentif à la complexité de la mise à mort, cartry en arrive à poser une hypothèse remarquable : "l'immolation, loin d'être un meurtre, est comme la reproduction du geste de la sage-femme, lorsque, coupant le cordon, elle sépare l'enfant d'avec son placenta". A ce moment du procès sacrificiel, la victime tient lieu d'équivalent du placenta. Tout se passe comme si, pour le sacrificateur, se trouvaient reproduits les termes d'une alternative : soit avoir à se séparer de "ce qui est plus soi-même que soi-même" (le placenta), soit suivre ce "compagnon" dans la mort. Cette dernière issue se trouverait réalisée dans d'autres cultures, où le sacrifiant se fait lui-même victime.

Dans cet ordre d'idées, on se souviendra que le paradigme mythique du sacrifice dogon est la naissance-mort du génie de l'eau Nommo, éviré au moment même où il est extrait du placenta originel : "le sacrificateur (...) repliant la.verge par-dessus le cordon, trancha l'ensemble. Ainsi Amma par cet acte séparait-il le sacrifié à la fois de son placenta et de son sexe" (2). Ce champ sẻmantique peut encore être étendu

2. GRIAULE, M. et DIETERLEN, G., Le Renard pẩe, 1965, p. 233. 
à des domaines voisins puisque, à propos du battage du fonio, les Dogon êtablissent aussi une équivalence symbolique entre le sang du sacrifice et le sang menstruel (3). On se demandera naturellement si, dans certains cas, 1'exclusion des femmes du sacrifice ne s'explique pas par cette "dette de sang", interprétée comme une participation naturelle à 1 'acte sacrificiel.

Chez les Thonga d'Afrique australe, en revanche, le sang n'est pas l'élément privilégié du sacrifice. Le questionnement qu'imposent les matériaux d'enquête récoltés par H.-A. Junod relève d'un tout autre ordre, à la fois culinaire et sociologique. Ce qui s'inscrit ici dans le corps dêpecé de 1 'animal lors des grands rites collectifs du mariage ou du deuil, c'est le corps même de la société, partagée entre le lignage patrilinéaire et 1'affiliation au lignage materne1. Le troupeau de chèvres constitue, chez les Thonga, une vêritable réserve sacrificielle, en marge du "capital" bovin utilisé, concurremment à d'autres biens, dans les circuits d'échange des femmes. Le porc, nouveau venu dans la culture thonga, est exclu quant à lui aussi bien des circuits économiques que des circuits religieux, et le mouton n'intervient que dans des circonstances exceptionnel1es. La lecture que je propose isole du vaste champ des offrandes (mhamba) un rite socio-religieux centra1 où les neveux utérins interviennent en qualité de "représentants" des ancêtres de leurs parents maternels ; alors qu'inversement 1a médiation des oncles maternels est dominante dans les rites privés (qu'ils soient ou non sacrificiels). Le sacrifice de fin de deuil dessine une topologie précise. L'utilisation du rumen de 1 'animal (qui relève du cru), comme les deux modes de cuisson distincts exigés des participants (le rôti et le bouilli), soulignent des différences de statut social et de résidence. Le dépeçage de la chèvre affirme et conjure tout à la fois une menace de dislocation de la société. C'est pourquoi les Thonga affirment que le sacrifice sanglant aide à "construire le village". C'est à un tout autre schème culinaire qu'obéit le sacrifice d'exorcisme des possédés, dont la fonction est de reconstituer $1^{\prime}$ unitê même du corps et de l'esprit, menacée par l'agression violente d'une puissance surnaturelle étrangère à la communauté lignagère. Le code introduit cette fois

3. GRIAULE, M., Dieu d'eau, Paris, 1948, pp. 175-183. 
I'absorption et le vomissement du sang cru, ainsi que la calcination des os dans un processus de restructuration du moi social.

Avec 1a communication de Nicole Grandin consacrée aux Arabes musulmans, nous inaugurons la seconde partie du programme que le Laboratoire s'était assigné, 1'extension de 1 a recherche à d'autres aires culturelles. L'auteur dégage la forme majeure du sacrifice musulman, rigoureusement codifié : celui que le pêlerin accomplit à La Mecque le 10 ăhul'hijja, et sa réplique réalisée à la même date dans I'ensemble de la comnunauté islamique, en dehors du lieu saint. Nicole Grandin évoque la polémique relative à l'interpétation des sacrifices de "rachat" qui ont lieu également à La Mecque ; elle rappelle enfin la persistance des formes populaires du sacrifice que les musulmans adressent aux esprits intermédiaires en đépit de la condamnation de l'islam officiel.

Nous abordons ensuite 1'Inde et la Mongolie. Comme le védisme, 1 'hindouisme populaire, qu'Olivier Herrenschmidt étudie à travers les pratiques rituelles de deux castes de pêcheurs en mer de la côte du Golfe du Bengale, accorde une position stratégique centrale au sacrifice. Mais il cesse d'être l'objet de spêculations savantes.

Herrenschmidt se trouve confronté à des déesses carnivores et à un dieu masculin végétarien. Le sacrifice sanglant est rêservé aux premières, les sakti et les ommoru. L'auteur situe son enquête au niveau de 1 'analyse structurale. Nul commentaire exégétique ne précise les différences internes existant entre ces deux catégories de déesses aux contours flous, et plus ou moins exigeantes. Mais il est clair que les ammoru, qui ne sont que des sakti relativement stabilisées dans l'ordre social (un peu à la manière de souverains redoutables) imposent -- au sens politique du terme -- des sacrifices bien circonscrits, alors que la voracité absolue des redoutables sakti, qu'il faut êloigner, embrasse un champ sacrificiel bien plus étendu. La pensée classificatoire s'attache ici à distinguer deux types de pratiques : le yata est la 
mise à mort par décolzation de boucs et de béliers, suivie d'une consommation alimentaire. Le korata implique des formes d'immolation très diversifiées mais toujours différentes de la précédente : 1'empalement, le tronçonnement, l'éventrement ou l'égorgement d'une victime choisie dans une série continue dont les termes sont rangés par ordre croissant d'impureté : 1'oeuf, le poussin, le coq, le bouc, le bélier, le porc, le buffle. La chair de 1'anima1, cette fois, n'est jamais mangée. Ces relations rituelles violentes impliquent enfin "qu'il n'y a au fond qu'une victime parfaite : 1 'homme lui-même".

C'est une problématique différente mais comparable qu'aborde Marine Carrin-Bouez dans un groupe austro-asiatique indien, constituant une unité endogame en marge du système des castes, les Santa1. Ici, 1'offrande par le prêtre de son propre sang domine la scène rituelle. Le naeke, prêtre du village, comme le ojha, "prêtre-devin", s'adonnent à ces pratiques. Dans le cas des ojha au moins, il est clair que le rituel est le substitut d'un sacrifice humain. Cet auto-sacrifice doit être mis en parallèle avec un schéma initiatique, qui semble relever du chamanisme : le corps du ojha, en effet, a èté dẻmembré puis reconstitué par son mâttre. Dans 1 'acte auto-sacrificie1, i1 rejoue apparement $1 a$ première partie de cette initiation puisqu'il morcelle son propre corps à partir de cinquante points. La façon dont il reconstitue ensuite sa force spirituelle ( $j i u$ ) demeure mystérieuse. Quant au sacrifice animal, il institue toujours une séparation (hatim, "diviser") : le sang aux dieux, la chair aux hommes. Selon 1'auteur, la formule atan, telan (reçu et accepté) qui s'applique à cette opération, relèverait de la sphère de l'échange. Mais la notion de hatim s'applique aussi au sacrifice d'exorcisme où l'on voit le ojha utiliser une volaille qu'il met en contact avec son propre corps, d'abord, celui du patient ensuite, avant d'extirper le mal par une morsure. Ne s'agirait-il pas, dans tous les cas, d'instaurer une coupure dans l'être vivant (homme ou animal), une déchirure dont la nature appelle un nouveau type d'élucidation ? L'auto-sacrifice du prêtre ojha, comme son initiation, mettent clairement en évidence la séquence symbolique morcellement-naissance et un mythe disqualifie précisêment les femmes comme sacrificateurs parce qu'elles s'étaient déclarées incapables de procéder au morcellement du boeuf. Marine Carrin-Bouez indique elle-même la piste qu'il conviendrait probablement de suivre lorsqu'elle souligne que "mise à 
mort et morcellement s'opposent ensemble au modèle de la reproduction" féminine. Le sacrificateur santal semble donner (voire se donner) la vie par la destruction d'une unité vivante déjà constituée (son propre corps parfois), que la femme ou la femelle a pour mission d'élaborer, avec de la chair et du sang. Que cette unité doive sans cesse être détruite, dans une apparence de partage, constitue 1 'une des énigmes majeures du sacrifice.

En tout état de cause, pas plus que dans l'hindouisme populaire, le sacrifice santal ne sous-tend l'ordre social de l'univers, comme c'est le cas dans la pensée indienne classique. On discerne même un singulier particularisme sociologique dans les pratiques sacrificielles des groupes de descendance. Les prescriptions rituelles qui les concernent renvoient au mythe d'origine propre à chacune de ces unités sociales, qui se différencient les unes des autres tout autant par le nom des divinités que par 1a manière de mettre à mort les victimes sacrificielles, ou par l'utilisation des ingrëdients. On découvre ainsi que 1a diversité inteme des systèmes sacrificiels est susceptible de marquer aussi le morcellement du corps social, en se référant à l'animal domestique, à la manière du code totémique, sans que naturellement, comme Lévi-Strauss 1 'avait bien vu, ces deux démarches ne puissent être confondues.

Roberte Hamayon aborde le domaine mongol avec les Bouriates des rives du lac Baĭkal. Elle introduit d'abord d'indispensables distinctions sociologiques. Le grand sacrifice tajlgan s'organise dans un cadre clanique, en liaison avec les ancêtres, mâ̂tres des montagnes ; le xerog s'effectue dans un cadre social restreint pour régler une "affaire" ; le dolig enfin est une vếritable "rançon" exigée pour sauver une vie humaine menacée par une très grave maladie. Les deux derniers rites requièrent toujours obligatoirement la présence du chaman; en outre ils s'adressent à "d'indignes anti-ancêtres" qui ont connu une mort anormale et se trouvent privés de descendance.

Le schéma sacrificiel est constánt. Il se présente sous la forme d'une cuisine rituelle particulièrement élaborée. La viande est bouillie ; celle qui se détache des os est consommée à 1a maison; seule 1a chair adhérant aux os est raclée et partagée avec les esprits ; les os eux-mêmes sont incinérés sur les pierres claniques. Enfin la dépouille (huraj), comportant d'un seul tenant la tête, 1'appareil respiratoire, 
les poumons et le coeur, est enfilée sur une perche. Deux traits complëmentaires des plus significatifs s'ajoutent à ces données de base lorsqu'on célèbre le plus individuel des trois rites, le dolig; le patient crache à trois reprises sur la victime et il inhale la vapeur que dẻgage la dëpouille huraj ; celle-ci sera conservée jusqu'à la guérison. C'est 1 'unique cas où 1 'animal sacrificiel apparaît comme un substitut du sacrifiant. Jadis la "rançon" exigée pouvait être une vie humaine et le rite prenait un caractère secret. On ne saurait conclure cependant, en termes maussiens, à une opposition entre des formes sacrificielles "religieuses", collectives ou publiques, et une forme "magique". La finalitẻ du rituel clanique xerog n'en diffère pas moins de celle du dolig. Alors que celui-ci rachète une vie humaine, celui-là reconstitue dans 1 'autre monde 1 'exacte réplique de 1 'animal sacrifié -- qui ne saurait être remplacé par une victime humaine. Roberte Hamayon conteste 1 'idêe que la viande, consommée par les hommes, serait en quelque façon une offrande alimentaire. Le traitement des os retiendra l'attention : leur incinération est 1 'opération médiatrice qui permet de reconstituer dans le monde des ancêtres l'exacte réplique de l'animal vivant; s'ils ne peuvent, avoir êté ni fracturês ni endomagés par le raclage de la viande, c'est que le squelette est 1 'armature de cette vêritable rêincarnation : la victime est transférée au troupeau surnaturel des ancêtres. L'efficacité symbolique de 1'incinération -- cet au-delà de la cuisine -- est précisément d'empêcher que cette transformation (qui reconstitue l'unité première de 1 'animal) n'ait lieu dans le domaine des vivants. On comprend que la victime soit toujours un animal intact, ni castré, ni marqué : tout signe d'appropriation s'opposerait à ce changement de 1ieu.

Roberte Hamayon montre de manière fort convaincante que la mise à mort symbolique du chaman, dont le corps est dépecé et la chair bouillie, l'assimile à une victime sacrificielle. Or le chaman est un "nouveau-né dont il faut couper le cordon ombilical". Très suggestive aussi est la comparaison entre le statut de $I^{\prime}$ animal, qui sera recomposé dans l'autre monde après dépeçage, et celui du candidat chaman, qui subit un sort analogue pour renaître ici-bas. Par ce biais au moins le schème sacrificiel présente, une fois de plus, des affinités avec. le fantasme de la naissance considérée corme une mort. De nouvelles questions se posent alors. La renaissance du chaman et la recomposition 
du corps de la victime animale -- ou de son double -- sont-elles conçues sur le mêne modèle ? Les catégories sacrificielles participentelles du symbolisme de la gestation ? Et pourquoi certains groupes bouriates désignent-ils expressément le grand rite clanique aux ancêtres par les termes "sacrifice au lieu d'enterrement du placenta" ?

Les animaux prélevês intacts, et non marqués, dans le cheptel de 1 'ẻleveur appartiennent à des espèces spécifiques : en principe, il s'agit d'animaux à museau chaud (chevaux et moutons) ; les bovins sont exclus, sans doute parce qu'un taureau mythique est $1^{\prime}$ ancêtre de l'ethnie, symbolisant son être par 1 'un des signes majeurs de 1 'avoir. Cette relation entre l'économique et le symbolique apparaît de plus en plus nettement comme $I^{\prime} u n$ des éléments-clé de l'analyse comparative. Certains rites concernant "des êtres en état d'infériorité sociale provisoire ou permanent" exigent cependant des chèvres, animaux à museau froid.

En marge du sacrifice proprement dit, Roberte Hamayon signale enfin 1 'existence d'animaux "consacrēs", qui sont le support vivant d'esprits. I1 est remarquable que 1 'on voit figurer au premier plan de cette nouvelle catégorie les bovins (exclus du sacrifice, on l'a vu), puis les chevaux, et exceptionnellement les caprins. Un parallèle africain s'impose immédiatement à 1 'esprit ; les Venda, dont l'animal sacrificiel est la chèvre, font d'un taureau noir la "représentation" des ancêtres du lignage patrilinéaire et d'une chèvre noire celle des ancêtres fêminins du lignage matrilinéaire (les Venda connaissent un système de double filiation) (4). Le régime de ces animaux-signes diffère chez les Venda et les Bouriates puisque les premiers ne les mettent jamais à mort et s'en servent comme d'autels vivants, alors que les seconds adoptent à leur égard des pratiques variables, mais qui ne sont jamais d'ordre sacrificiel. L'opposition entre ces deux usages rituels de l'animal domestique est clairement établie chez les Bouriates : "Alors que les sacrifiés montent des hommes vers les esprits, les consacrés représentent une descente des esprits vers les hommes".

Voici qu'émerge, aux frontières du champ sacrificiel, 1'usage ri-

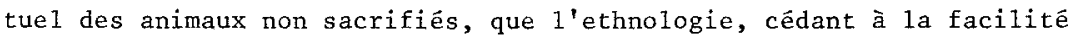

4. STAYT, The Bavenda, Londres, 1968. 
thêorique, qualifie volontiers de "sacrés".

Cette expression, qui nous vient en ligne droite de Durkheim, à travers une tradition suspecte, évite de poser le problème général, évoqué tout au long de ces essais : le statut socio-religieux de l'animal domestique, vivant dans la proximité de l'homme, à portée de main. La valeur économique souvent arbitraire de cet objet vivant. asservi ne saurait à elle seule rendre compte de la question lancinante du sacrifice. En tout état de cause celui-ci ne se réduit pas à son apparence d'offrande alimentaire, pas plus qu'à un rite de passage utilisant la victime comme le lieu d'une obscure "sacralisation" d"un être vivant "profane" en vue d'assurer la communication avec les dieux. Le dépeçage et la cuisine rituelle, quelle que soit la diversité des finalités poursuivies, renvoient toujours à un registre symbolique où le don de nourriture est souvent totalement absent. On ajoutera cependant aussitôt que le "cannibalisme" de certaines divinités qui exigent des impositions de sang ou des rançons pose des problèmes d'interprétation particulièrement complexes. Quel est ce fantasme qui traverse ces cultures indiennes qui opposent radicalement divinités carnivores et végétariennes ?

Les itinéraires variés, indiqués par ce nouvel échantillonage, ne permettent évidemment pas encore de conclusion d'ensemble. Ils suscitent de nombreuses questions et parfois de nouvelles hypothèses de recherche. Nous consacrerons un troisième cahier à de nouvelles ouvertures sociologiques, économiques et symboliques de cette recherche comparative, dont on aperçoit déjà qu'elle met radicalement en cause le schéma maussien. 